\title{
Coronary endarterectomy for diffusely diseased coronary artery: An ace in the hole in coronary artery surgery
}

Kosaku Nishigawa, MD, ${ }^{\mathrm{a}, \mathrm{b}}$ Toshihiro Fukui, MD, PhD, ${ }^{\mathrm{a}, \mathrm{b}}$ Jun Takaki, MD, ${ }^{\mathrm{a}}$ and Shuichiro Takanashi, MD, PhD ${ }^{\mathrm{b}, \mathrm{c}}$

Video clip is available online.

\begin{abstract}
Feature Editor's Introduction-Coronary thromboendarterectomy represents one of the most technically challenging procedures for the coronary surgeon. Although in most cases, careful selection of the anastomotic site (eventually with the help of intraoperative imaging) may avoid the need for endarterectomy, there are patients and target vessels for which the surgeon's expertise with the procedure makes the difference between a suboptimal operation and a good result that will benefit the patients for the years to come. As is often the case in surgery, attention to details is key, and in this issue of JTCVS Techniques, the group from Kumamoto University Hospital provide superb guidance on the various steps of this challenging but important procedure. I'm sure that the readers of JTCVS Techniques will enjoy reading this piece as much as I did.
\end{abstract}

\section{Mario Gaudino, MD, PhD}

In the late 1950s, coronary endarterectomy (CE) was successfully introduced as a surgical option for angina pectoris. ${ }^{1,2}$ However, initially many cardiac surgeons were reluctant to perform this procedure, because the patients who underwent $\mathrm{CE}$ were at higher risk of surgical morbidity, such as perioperative myocardial infarction (POMI), and mortality. Moreover, coronary artery bypass grafting $(\mathrm{CABG})$ was introduced in the late $1960 \mathrm{~s}^{3}$ and rapidly became the gold standard for surgical coronary revascularization. Therefore, CE is a historical and unfamiliar procedure for most modern cardiac surgeons.

\footnotetext{
From the ${ }^{\text {a Department }}$ of Cardiovascular Surgery, Kumamoto University Hospital, Kumamoto, Japan; 'bepartment of Cardiovascular Surgery, Sakakibara Heart Institute, Tokyo, Japan; and ${ }^{\mathrm{c}}$ Department of Cardiac Surgery, Kawasaki Saiwai Hospital, Kawasaki, Japan.

Received for publication March 10, 2021; accepted for publication March 11, 2021; available ahead of print March 21, 2021.

Address for reprints: Toshihiro Fukui, MD, PhD, Department of Cardiovascular Surgery, Kumamoto University Hospital, 1-1-1 Honjo, Chuo-ku, Kumamoto, 8608556, Japan (E-mail: tfukui.cvs@gmail.com).

JTCVS Techniques 2021;10:133-7

2666-2507

Copyright (C) 2021 The Author(s). Published by Elsevier Inc. on behalf of The American Association for Thoracic Surgery. This is an open access article under the CC BY-NC-ND license (http://creativecommons.org/licenses/by-nc-nd/4.0/).

https://doi.org/10.1016/j.xjtc.2021.03.018
}

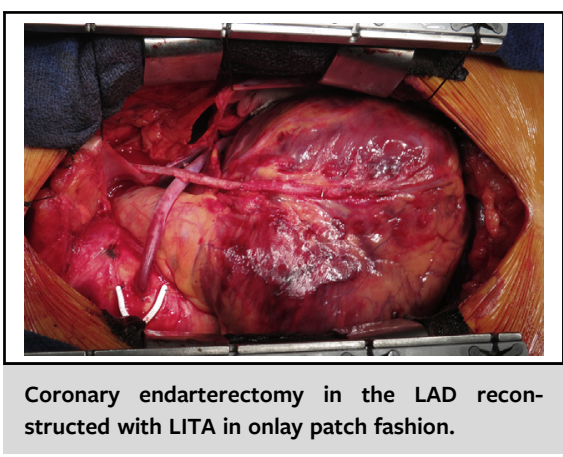

\begin{abstract}
CENTRAL MESSAGE
Coronary endarterectomy can be an effective and ultimate surgical alternative for diffusely diseased coronary arteries.
\end{abstract}

See Commentary on page 138.

\section{RATIONALE AND INDICATIONS}

Since the introduction of drug-eluting stents (DES), the indication for percutaneous coronary intervention (PCI) has been expanded to multivessel and left main diseases, for which CABG was conventionally indicated. This trend accelerated remarkably after the emergence of second-generation DES. Thereafter, the number of CABG procedures has been decreasing, and the complexity of coronary artery disease (CAD) referred for CABG has been increasing. Based on the findings of several randomized studies, the latest European Society of Cardiology/European Association for Cardio-Thoracic Surgery Guidelines on myocardial revascularization recommends CABG over PCI for highly complex CADs, such as left main or triple-vessel diseases with intermediate or high SYNTAX scores. ${ }^{4}$ Complex CADs frequently include diffuse lesions complicated by severe calcifications or narrow segments. Diffusely diseased coronary arteries with or without severe calcification are challenging for coronary interventionists and cardiac surgeons. It is difficult to perform distal anastomosis to the healthy segment of the target coronary artery, and simple anastomosis distal to the diffusely diseased segment of the left anterior descending artery (LAD) cannot achieve complete revascularization of the myocardial territories supplied by the side branches (such as septal perforators and diagonal branches) affected by the diffuse lesions. ${ }^{5}$ For these cases, CE can be an effective surgical alternative for complete myocardial 
revascularization. Therefore, we need to reconsider the effectiveness and significance of $\mathrm{CE}$ in this era of new-generation DES, in which the complexity of CAD in CABG candidates has been increasing.

Patients with diffusely diseased coronary artery cannot achieve the benefits of $\mathrm{CE}$ when the myocardium supplied by the target coronary artery has no viability because of previous myocardial infarction. We do not perform CE for the diffusely diseased LAD when the viability in the anteroseptal region is no longer proven by preoperative evaluation, such as myocardial scintigraphy.

Moreover, we can apply the concepts and techniques of $\mathrm{CE}$ to in-stent restenosis (ISR) involving side branches in the LAD. Once ISR has occurred in patients with "full metal-jacketed" LAD, the anteroseptal region perfused by the side branches suffered from myocardial ischemia (stent jail). For these cases, we cannot obtain complete myocardial revascularization of the entire anteroseptal region by simple distal anastomosis, and the LAD is often ungraftable because of multiple stents implanted in its distal portion. Our group reported a successful series of 12 patients who underwent $\mathrm{CE}$ with concomitant stent removal and onlay patch reconstruction using the left internal thoracic artery (LITA) for long-segment ISR in the LAD. ${ }^{6}$

\section{TECHNIQUES OF CE}

Two methods are available for CE: closed (traction) and open (direct-vision) endarterectomies. Although closed endarterectomy requires only limited arteriotomy and is simpler, incomplete removal of atherosclerotic plaques and shearing-off of the plaques in the side branches (called "snow-plow" effect) is a major concern. Moreover, this procedure carries the risk of coronary artery dissection and subsequent intimal flap formation that can cause distal occlusion. These complications may be associated with POMI and surgical mortality, which were initially reported to worsen outcomes of CE. In contrast, open endarterectomy facilitates complete removal of the atherosclerotic lesion under direct vision, although it requires a technically demanding and time-consuming procedure. We consider that complete removal of atherosclerotic plaques is mandatory to achieve complete revascularization and superior graft patency. Nishi and colleagues reported that the patency rate of open endarterectomy with onlay patch grafting was superior to that of traction endarterectomy $(89.1 \%$ vs $81.0 \%$ at 5 years, $P<.001) .{ }^{7}$ Currently, open endarterectomy is preferred to closed endarterectomy for removal of severe atheromatous plaques, especially for the LAD. Moreover, several procedures have been reported as reconstruction methods for endarterectomized coronary arteries, including saphenous vein patching with or without LITA grafting or onlay patch grafting using the LITA. However, onlay patch grafting using the LITA provides a superior patency rate, because the greater part of the lumen of the reconstructed LAD consists of healthy LITA intima.

We have also consistently performed direct-vision endarterectomy with onlay-patch reconstruction using the LITA for diffusely diseased LAD. ${ }^{8}$ Coronary arteriotomy is begun in the mid-portion of the LAD and extended proximally and distally. The length of the incision depends on the length of atherosclerotic lesion. The atheromatous core is carefully dissected from the adventitia with fine forceps and a spatula, especially when we perform $\mathrm{CE}$ with concomitant stent removal. Endarterectomy is continued into the side branches as long as the spatula reaches and the distal end of the intima of the side branches is pulled out. The proximal end of the atheromatous core is divided to avoid removing the most proximal stenotic lesion, to prevent competitive flow through the native coronary artery. The distal end of the atheromatous core is sharply divided at the intact intima, and the divided intima is tacked with 80 polypropylene sutures to prevent flap formation. We carefully remove the residual medial tissue on the endovascular surface in the LAD using a spatula and wash out the surface with saline solution. Thereafter, the skeletonized LITA is incised to match the length of the LAD arteriotomy and anastomosed to the LAD with several 8-0 polypropylene sutures in onlay-patch fashion (Video 1). After completion of the anastomosis, we routinely use transit-time flowmetry to evaluate the graft and anastomosis according to the same criteria for conventional anastomosis.

Performing CE in combination with on-pump or offpump CABG is still controversial. Because CE is complex and time-consuming, surgeons may be reluctant to perform CE with an off-pump procedure. However, Shapira and colleagues ${ }^{9}$ demonstrated that prolonged cardiopulmonary bypass time was an independent predictor of POMI. Open endarterectomy via a long arteriotomy is a timeconsuming procedure, and $\mathrm{CE}$ with off-pump $\mathrm{CABG}$ avoids longer cardiopulmonary bypass and cross-clamp times. We usually perform $\mathrm{CABG}$ with concomitant $\mathrm{CE}$ using an

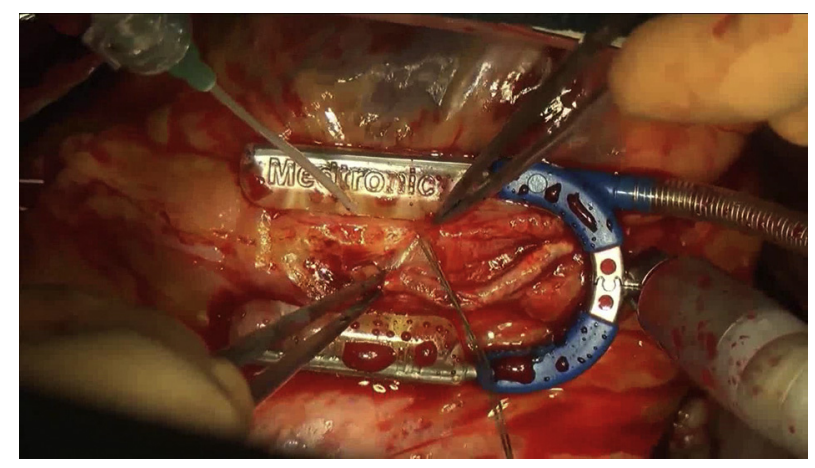

VIDEO 1. Intraoperative video of open endarterectomy and reconstruction with the left internal thoracic artery. Video available at: https:// www.jtcvs.org/article/S2666-2507(21)00265-0/fulltext. 

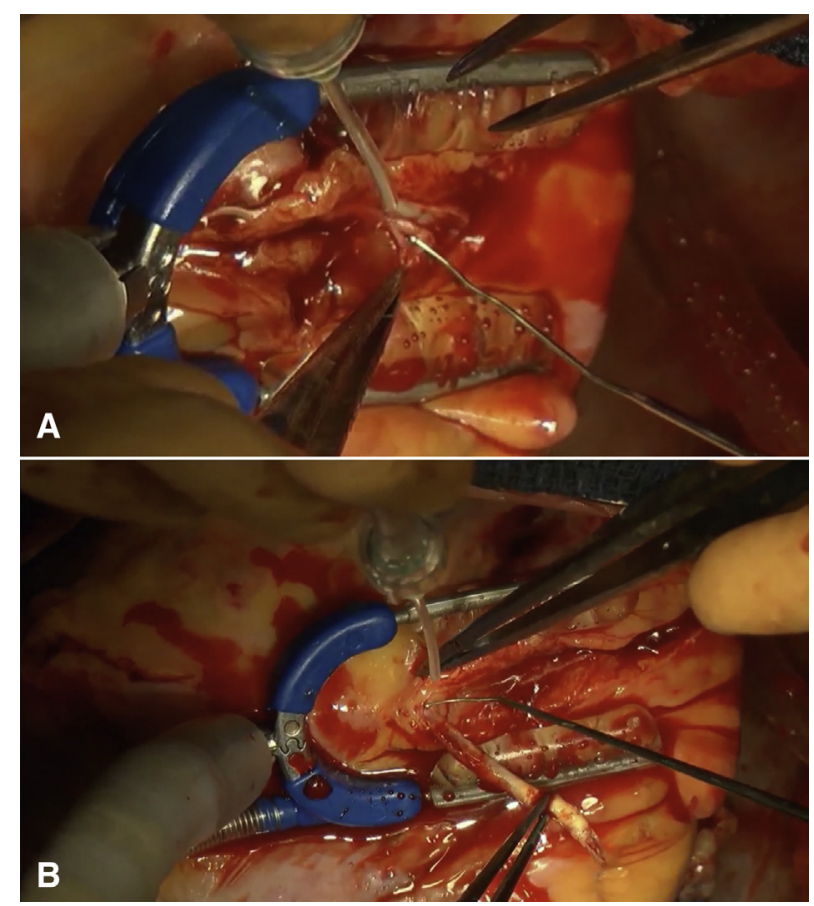

FIGURE 1. Intraoperative photographs of removing the atheromatous core. A, The endarterectomy plane is easily identified. B, The calcified atheromatous core naturally comes off from the adventitia on the beating heart.

off-pump technique whenever feasible, to avoid longer cardiopulmonary bypass and cross-clamp times. ${ }^{8}$ Moreover, when we perform $\mathrm{CE}$ for the LAD on the beating heart with an off-pump procedure, we can easily detect the endarterectomy plane and remove the atheromatous plaques, because the hard atheromatous core naturally comes off from the soft adventitia along with the heartbeat (Figure 1). In our experience, a diffusely diseased LAD can tolerate longer ischemic times during anastomosis because sufficient collaterals would have developed; therefore, patients undergoing CE rarely require on-pump conversion. Indeed, there are several reports describing off-pump CABG with concomitant $\mathrm{CE}$ with a low conversion rate $(0 \%-8 \%))^{10-12}$ Moreover, CE with on-pump beating $\mathrm{CABG}$ can be the preferable alternative for the majority of cardiac surgeons who are unfamiliar with off-pump CE.

\section{PITFALL AND POSTOPERATIVE ANTICOAGULATION}

Acute thrombosis in the reconstructed LAD is one of the most disastrous complications after CE that causes POMI in the broad anteroseptal region. This complication is caused when the lack of endothelium in the endarterectomized portion exposes the subendothelial tissue to the

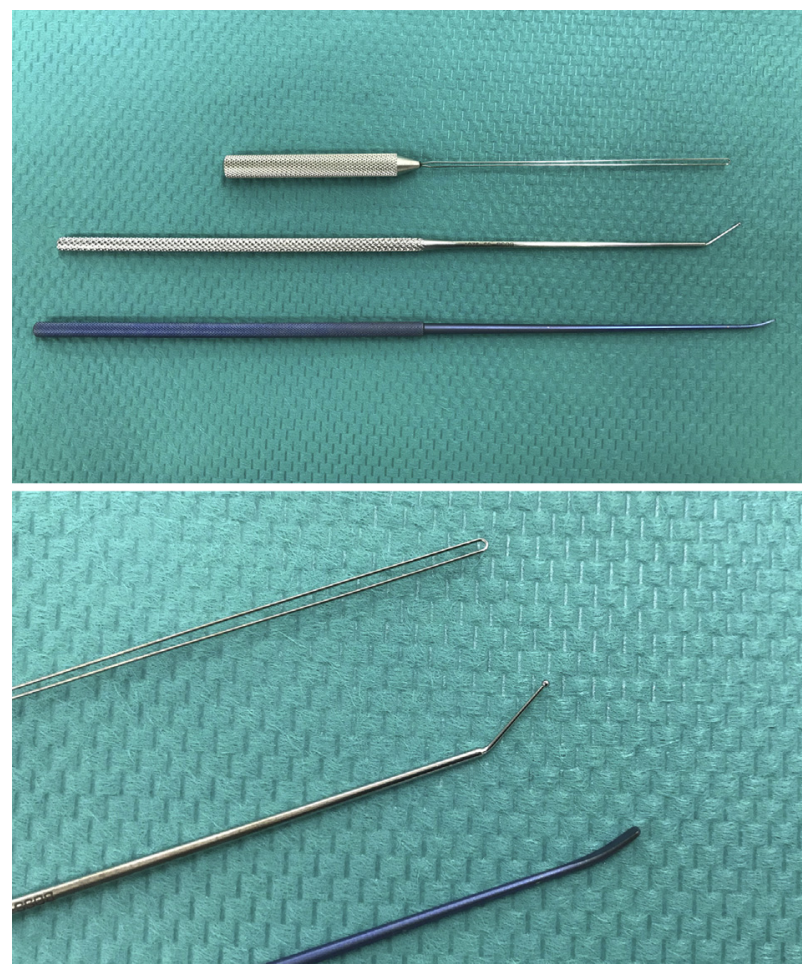

FIGURE 2. Surgical instruments for coronary endarterectomy at our institution.

bloodstream, thereby triggering the coagulation cascade. Therefore, strict anticoagulation is indispensable to prevent thrombosis in endarterectomized vessels. The intraoperative anticoagulation protocol at our institution is as follows: the activated clotting time (ACT) is maintained for 450 to 500 seconds during surgery with systemic heparinization and reversed with minimum dose of protamine at the end of surgery (target ACT is approximately 200 seconds). Postoperatively, continuous intravenous infusion of low-molecular-weight heparin (5000 units/day) is initiated after chest tube output has settled and continued until warfarin is effective. Low-dose aspirin (100 mg/day), clopidogrel $(75 \mathrm{mg} /$ day), and warfarin (titrated to a target international normalized ratio of 2.0-2.5) are started after the initiation of oral intake. Our previous studies demonstrated that the diameter of the reconstructed LAD decreased to match that of the LITA in follow-up angiography performed 1 year after surgery (vascular remodeling) (Figure 2). ${ }^{13}$ We further evaluated the lumen of the reconstructed LAD with intravascular optical coherence tomography and found that the endarterectomized LAD was completely endothelialized within 1 year after surgery. ${ }^{8}$ Once endothelialization of the reconstructed vessel lumen is attained, anticoagulant agents are no longer necessary. We discontinue warfarin and clopidogrel at 3 months and 1 year after surgery, respectively, but aspirin is continued indefinitely. 

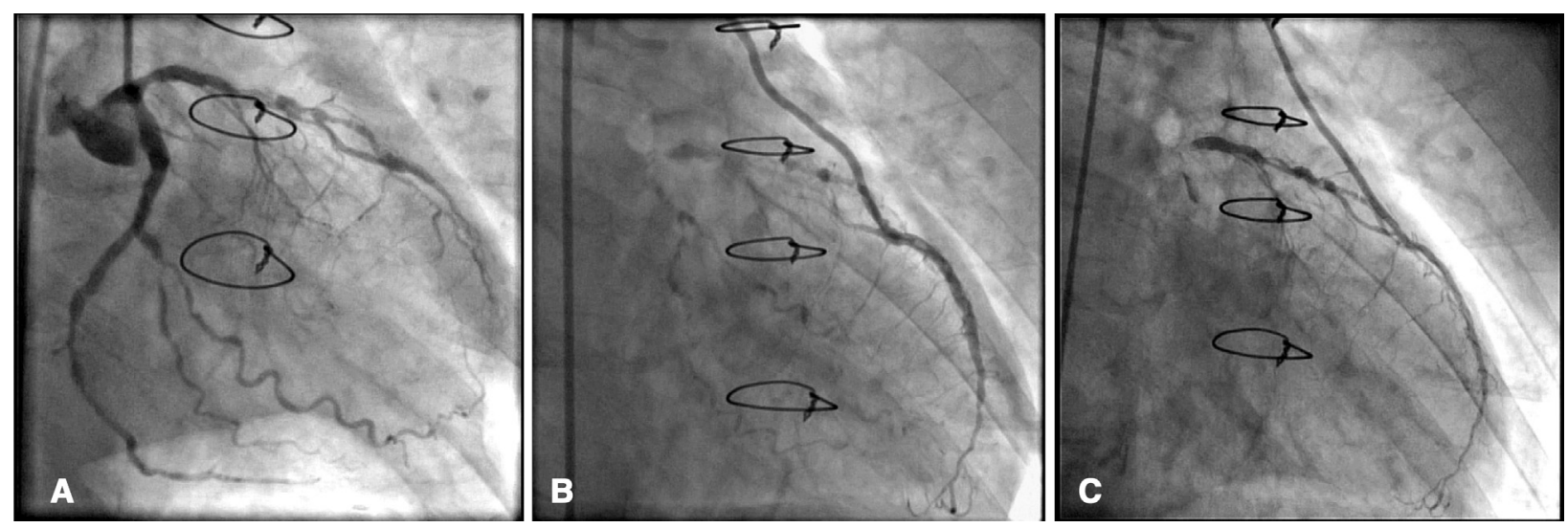

FIGURE 3. Representative angiographic images obtained preoperatively (A), early postoperatively (B), and at 1 year postoperatively (C).

\section{SURGICAL RESULTS OF CE}

Between September 2004 and June 2017, 233 patients underwent $\mathrm{CABG}$ with concomitant $\mathrm{CE}$ for the diffusely diseased LAD at Sakakibara Heart Institute, Tokyo. Of these, 24 patients $(10.3 \%)$ underwent coronary stent removal for ISR in the LAD. The mean patient age was $65.9 \pm 9.5$ year and 200 patients $(85.8 \%)$ were male. The mean number of distal anastomoses was $4.5 \pm 1.3$, and the mean length of the incision on the LAD was $6.1 \pm 1.7 \mathrm{~cm}$. Two hundred thirty-two patients $(99.6 \%)$ underwent surgery with an off-pump procedure, and 16 $(6.9 \%)$ required on-pump conversion. The mean operating time was $358 \pm 71$ minutes. The median postoperative creatine kinase-MB level was $32 \mathrm{IU} / \mathrm{L}$ (interquartile range, 21 to $57 \mathrm{IU} / \mathrm{L}$ ). Postoperatively, stroke occurred in 8 patients $(3.4 \%)$, and deep sternal wound infection was seen in 5 patients $(2.3 \%)$. Operative mortality was $0.9 \%$ ( 2 of 233 ). The early and 1-year angiographic patency rates of the reconstructed LAD were $91 . \%$ and $97.1 \%$, respectively (Figure 3). The freedom from all-cause death at 5 years was $90 \%$, and that from major adverse cardiac and cerebrovascular events was $73.1 \%$.

In conclusion, although technically demanding and unfamiliar to many cardiac surgeons, we should reconsider the significance of CE for diffusely diseased coronary arteries in this era of increasing numbers of highly complex CAD with severe calcification or diffuse lesions, in association with the progressive application of PCI using newgeneration DES.

\section{FUTURE RECOMMENDATIONS FOR RESEARCH}

As mentioned above, there are several reports describing the clinical and angiographic outcomes of CE for diffusely diseased coronary arteries. However, few studies have demonstrated the results of functional evaluations after CE. Angiographic patency does not necessarily mean sufficient blood supply to the myocardium perfused by the revascularized coronary artery. Functional studies using cardiac nuclear medical examination, such as myocardial perfusion scintigraphy, or evaluation of wall motion in the anteroseptal region using speckle tracking or dobutaminestress echocardiography, are needed to elucidate the impact of $\mathrm{CE}$ on myocardial perfusion and cardiac function.

To date, there are no definitive guidelines or protocols for postoperative antiplatelet or anticoagulation treatment for patients who have undergone CE. Recently, although several meta-analyses have demonstrated that dual antiplatelet therapy is associated with a significant reduction in vein graft occlusions after $\mathrm{CABG},{ }^{14,15}$ no obvious effect was proven in arterial grafts. Myers and colleagues reported that medication administered postoperatively (warfarin or clopidogrel) did not significantly affect late mortality after extensive LAD endarterectomy. ${ }^{16}$ Similarly, Russo and colleagues ${ }^{17}$ reported that in patients who underwent CE, no significant difference in midterm clinical outcomes was observed between those treated with single- versus dualplatelet therapy. However, because angiographic analyses were not performed in both studies, the impact of postoperative antiplatelet/anticoagulation regimen on the patency of reconstructed vessels after $\mathrm{CE}$ is still unclear. In our opinion, optimal postoperative medication following $\mathrm{CE}$ should be different from that of conventional CABG, because $\mathrm{CE}$, unlike conventional distal anastomosis, requires direct manipulation of the diseased portion of the target coronary artery. Therefore, further investigations are needed to determine the optimal medication after CE for the prevention of acute thrombosis and improvement of graft patency.

\section{Conflict of Interest Statement}

The authors reported no conflicts of interest.

The Journal policy requires editors and reviewers to disclose conflicts of interest and to decline handling or reviewing manuscripts for which they may have a conflict of interest. The editors and reviewers of this article have no conflicts of interest. 


\section{References}

1. Bailey CP, May A, Lemmon WM. Survival after coronary endarterectomy in man. JAMA. 1957;164:641-6.

2. Longmire WP Jr, Cannon JA, Kattus AA. Direct-vision coronary endarterectomy for angina pectoris. N Engl J Med. 1958;259:993-9.

3. Favaloro RG. Saphenous vein autograft replacement of severe segmental coronary artery occlusion: operative technique. Ann Thorac Surg. 1968;5:334-9.

4. Sousa-Uva M, Neumann FJ, Ahlsson A, Alfonso F, Banning AP, Benedetto U, et al; ESC Scientific Document Group. 2018 ESC/EACTS guidelines on myocardial revascularization. Eur J Cardiothorac Surg. 2019;55:4-90.

5. Nishigawa K, Fukui T, Takanashi S. Coronary endarterectomy for the diffusely diseased coronary artery. Gen Thorac Cardiovasc Surg. 2014;62:461-7.

6. Nishigawa K, Fukui T, Takanashi S. Off-pump coronary endarterectomy with stent removal for in-stent restenosis in the left anterior descending artery. Interact Cardiovasc Thorac Surg. 2015;21:594-7.

7. Nishi H, Miyamoto S, Takanashi S, Minamimura H, Ishikawa T, Kato Y, et al. Optimal method of coronary endarterectomy for diffusely diseased coronary arteries. Ann Thorac Surg. 2005;79:846-52; discussion 852-3.

8. Nishigawa K, Fukui T, Yamazaki M, Takanashi S. Ten-year experience of coronary endarterectomy for the diffusely diseased left anterior descending artery. Ann Thorac Surg. 2017;103:710-6.

9. Shapira OM, Akopian G, Hussain A, Adelstein M, Lazar HL, Aldea GS, et al. Improved clinical outcomes in patients undergoing coronary artery bypass grafting with coronary endarterectomy. Ann Thorac Surg. 1999;68:2273-8.

10. Eryilmaz S, Inan MB, Eren NT, Yazicioglu L, Corapcioğlu T, Akalin H. Coronary endarterectomy with off-pump coronary artery bypass surgery. Ann Thorac Surg. 2003; 75:865-9.
11. Vohra HA, Kanwar R, Khan T, Dimitri WR. Early and late outcome after off-pump coronary artery bypass graft surgery with coronary endarterectomy: a single-center 10-year experience. Ann Thorac Surg. 2006;81: 1691-6.

12. Takahashi M, Gohil S, Tong B, Lento P, Filsoufi F, Reddy RC. Early and midterm results of off-pump endarterectomy of the left anterior descending artery. Interact Cardiovasc Thorac Surg. 2013;16:301-5.

13. Shimokawa T, Manabe S, Fukui T, Takanashi S. Remodeling of reconstructed left anterior descending coronary arteries with internal thoracic artery grafts. Ann Thorac Surg. 2009;88:54-7.

14. Deo SV, Dunlay SM, Shah IK, Altarabsheh SE, Erwin PJ, Boilson BA, et al. Dua anti-platelet therapy after coronary artery bypass grafting: is there any benefit? A systematic review and meta-analysis. J Card Surg. 2013;28:109-16.

15. Nocerino AG, Achenbach S, Taylor AJ. Meta-analysis of effect of single versus dual antiplatelet therapy on early patency of bypass conduits after coronary artery bypass grafting. Am J Cardiol. 2013;112:1576-9.

16. Myers PO, Tabata M, Shekar PS, Couper GS, Khalpey ZI, Aranki SF. Extensive endarterectomy and reconstruction of the left anterior descending artery: early and late outcomes. J Thorac Cardiovasc Surg. 2012;143:1336-40.

17. Russo M, Nardi P, Saitto G, Bovio E, Pellegrino A, Scafuri A, et al. Single versus double antiplatelet therapy in patients undergoing coronary artery bypass graft ing with coronary endarterectomy: mid-term results and clinical implications. Interact Cardiovasc Thorac Surg. 2017;24:203-8.

Key Words: coronary endarterectomy, onlay patch grafting, CABG 